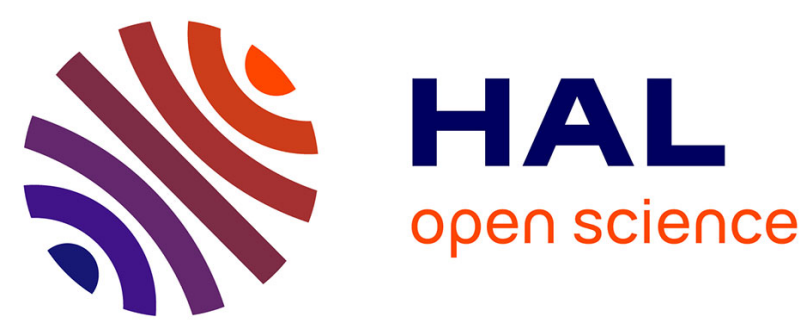

\title{
L'utilisation des techniques d'hybridation in situ et de clonage de séquences d'ADN pour l'étude des chromosomes chez les végétaux
}

Jane Hutchinson, Christiane Fauron

\section{- To cite this version:}

Jane Hutchinson, Christiane Fauron. L'utilisation des techniques d'hybridation in situ et de clonage de séquences d'ADN pour l'étude des chromosomes chez les végétaux. Agronomie, 1984, 4 (10), pp.957-966. hal-00884599

\section{HAL Id: hal-00884599 \\ https://hal.science/hal-00884599}

Submitted on 1 Jan 1984

HAL is a multi-disciplinary open access archive for the deposit and dissemination of scientific research documents, whether they are published or not. The documents may come from teaching and research institutions in France or abroad, or from public or private research centers.
L'archive ouverte pluridisciplinaire HAL, est destinée au dépôt et à la diffusion de documents scientifiques de niveau recherche, publiés ou non, émanant des établissements d'enseignement et de recherche français ou étrangers, des laboratoires publics ou privés. 


\title{
L'utilisation des techniques d'hybridation in situ et de clonage de séquences d'ADN pour l'étude des chromosomes chez les végétaux
}

Jane HUTCHINSON (1) \& Christiane FAURON

Cytogenetics Department, Plant Breeding Institute, Trumpington, Cambridge, CB2 2LQ, Grande-Bretagne

RÉSUMÉ

\begin{abstract}
Cet article présente une revue des méthodes utilisées pour le clonage de séquences d'ADN dans des vecteurs comme des plasmides, cosmides et le phage lambda, en même temps que la technique de localisation des séquences clonés sur les chromosomes en utilisant l'hybridation in situ.

Il donne une description détaillée de l'application de ces techniques à l'étude de la structure, l'organisation et
\end{abstract} l'identification des chromosomes chez les plantes.

Mots clés additionnels : Plasmide, cosmide, phage lambda, identification des chromosomes, organisation des séquences.

The methods used for cloning DNA sequences in plasmid, cosmid and phage lambda vectors are reviewed, together with the technique for localising the cloned sequences on chromosomes using in situ hybridization. Applications of these techniques to the study of the structure, organization and identification of chromosomes in plants are described in detail.

Additional key words : Plasmid, cosmid, phage lambda, chromosome identification, sequence arganisation.

Un programme d'amélioration des plantes faisant appel à l'ingéniérie chromosomique nécessite l'identification individuelle des chromosomes. Traditionnellement, on utilise des caractères tels que la longueur et la position du centromère surtout chez les espèces diploïdes ; dans le cas d'espèces polyploïdes comme le blé tendre où les chromosomes sont difficilement reconnaissables par leur taille, on a employé des lignées aneuploïdes ou porteuses de chromosomes marqués cytologiquement, notamment des chromosomes télocentriques. Plus récemment, les techniques de coloration différentielle ont apporté quelques informations supplémentaires. L'hybridation in situ à partir de séquences clonées permet aujourd'hui de compléter ces méthodes.

\section{LA TECHNIQUE D'HYBRIDATION IN SITU}

La technique d'hybridation in situ est basée sur le principe de la formation de duplexes ARN-ADN ou

(1) Adresse actuelle : Department of Biological Sciences, Manchester Polytechnic, Oxford Road, Manchester, Grande-Bretagne.
ADN-ADN où l'une des molécules est immobilisée dans la cellule et l'autre utilisée comme sonde moléculaire pour la localiser. La méthodologie, développée à la fin des années 60 (GALL \& PARDUE, 1971), a été largement appliquée à un grand nombre d'organismes. Depuis cette date, de nombreuses mises au point concernant cette technique et ses applications ont été publiées (JONES, 1973 ; WIMBER \& STEFFENSEN, 1973 ; PARDUE \& GALL, 1975 ; WOBUS, 1976 ; HUTCHINSON et al., 1981, etc...). Quelques modifications mineures ont été introduites en vue de recherches particulières.

Dans le cas des plantes, on prépare tout d'abord sur une lame des étalements de chromosomes mitotiques ou méiotiques : des extrémités de racines prétraitées (APPELS et al., 1978) ou des anthères (HUTCHINSON et al., 1980) sont fixées dans l'alcool acétique $3: 1$, puis écrasées entre lame et lamelle dans l'acide acétique à 45 p. 100. Après l'écrasement, les préparations sont traitées à la neige carbonique ou à l'azote liquide ce qui permet de séparer lame et lamelle, sans perdre les celllules qui restent fixées sur la lame. Les préparations sont ensuite déshydratées à l'alcool éthylique 
absolu et séchées à l'air. A ce stade, tout ARN endogène qui pourrait interférer avec l'hybridation est détruit par traitement des préparations à l'ARNase (GALL \& PARDUE, 1971). L'étape suivante consiste à dénaturer l'ADN chromosomique par traitement soit à l'acide, soit à l'alcali, soit à la chaleur (voir revues déjà citées). Pour les chromosomes des plantes, 2 traitements sont couramment utilisés : la dénaturation avec $\mathrm{HCl}$ 0,2 N (PEACOCK et al., 1977) et la dénaturation par action combinée de la chaleur et de la formamide (HUTCHINSON et al., 1980). On peut également appliquer simultanément les 2 traitements (GERLACH et al., 1982).

On prépare ensuite des sondes moléculaires radioactives. Plusieurs méthodes peuvent être employées (voir revues) : pour identifier les chromosomes des plantes, la meilleure méthode consiste à utiliser l'ARN complémentaire (marqué au tritium) des séquences d'ADN. Cet ARN est obtenu à l'aide de l'enzyme ARN polymérase, en présence de ${ }^{3} \mathrm{H}$ nucléotides triphosphates (GALL \& PARDUE, 1971). La sonde ${ }^{3} \mathrm{H}-\mathrm{ARN}$ est préparée dans une solution de $6 \times \mathrm{SSC}$, puis diluée de moitié avec de la formamide avant hybridation. La solution d'hybridation est donc de $3 \times \mathrm{SSC}-50 \mathrm{p}$. 100 formamide. Un volume de 6 à $8 \mu \mathrm{l}$ de cette solution est placé sur la préparation, qui est alors recouverte d'une lamelle, puis lavée à l'acide $(\mathrm{HCl} 0,2 \mathrm{~N})$ et scellée. La durée et la température requises pour l'hybridation dépendent de la complexité de la séquence, de la présence de répétitions et de l'activité spécifique de la sonde (SZABO et al., 1977). En général, l'identification des chromosomes des plantes demande un temps d'hybridation d'environ $16 \mathrm{~h}$ à une température de $40 \pm 1{ }^{\circ} \mathrm{C}$ (GERLACH et al., 1982). Tout excès de sonde est alors éliminé par une série de lavages très méticuleux et par d'autres traitements à l'ARNase (HUTCHINSON et al., 1981; GERLACH et al., 1982). Les lames sont ensuites déshydratées et séchées à l'air avant d'être recouvertes par une émulsion photosensible. Le temps d'exposition des autoradiographies dépend du degré répétitif des séquences et de l'intensité du marquage de la sonde. Les préparations sont donc développées et les essais sont répétés autant de fois que cela est nécessaire pour obtenir l'exposition optimale. Après le développement, on fixe puis on colore les préparations, le plus souvent avec une solution aqueuse à $10 \mathrm{p}$. 100 de Giemsa. La présence de grains d'argent dans l'émulsion photographique indique la localisation des séquences utilisées comme sonde.

Avant que le clonage moléculaire des séquences d'ADN de plantes n'ait été mis au point, la plupart des séquences utilisées pour l'hybridation in situ des chromosomes de plantes étaient des séquences répétitives isolées, séparées de l'ADN principal grâce à leurs propriétés physiques. Une fraction de l'ADN hautement répétitif nommé $\mathrm{ADN}$ Cot $10^{-2}$ était séparée du reste de l'ADN sur colonne d'hydroyapatite grâce à sa renaturation très rapide après dénaturation (Cot $=$ moles nucléotide $1 \times$ temps d'incubation en s). C'est ainsi que les fractions Cot isolées à partir des chromosomes de Secale cereale (APPELS et al., 1978), Luzula purpurea (RAY \& VENKETESWARAN, 1979), Triticum aestivum (GERLACH \& PEACOCK, 1980) ont été réhybridées à leurs chromosomes respectifs. L'exa- men des chromosomes met en évidence des régions d'hybridation très spécifiques mais aussi des sites d'hybridation plus dispersés. Etant donné que l'ADN $\mathrm{Cot}^{-2}$ représente un mélange de familles de séquences répétitives sans relation les unes avec les autres, il n'est pas possible dans ce cas de connaître la localisation d'une séquence particulière ; chaque site pouvant être attribué à plus d'une famille (GERLACH \& PEACOCK, 1980 ; HUTCHINSON \& LONSDALE, 1982). On a donc, par la suite, cherché à isoler certains composants de l'ADN $\operatorname{Cot}^{-2}$, ce qui a été possible car certaines familles de séquences hautement répétitives appelées $\mathrm{ADN}$ satellite présentent une composition en bases très particulière et peuvent être séparées du reste de l'ADN par centrifugation en gradients de densité. De tels ADN satellites ont été isolés à partir de Scilla sibirica (TIMMIS et al., 1975 ; DEUMLING \& GreIlHuber, 1982), Vicia faba (TIMMIS et al., 1975), Triticum aestivum et Hordeum vulgare (GERLACH et al., 1978 ; DENNIS et al., 1980) et Zea mays (PEACOCK et al., 1981b) et localisés sur les chromosomes par hybridation in situ. Cependant, comme pour l'ADN $\mathrm{Cot}^{-2}$, l'ADN satellite, bien que moins hétérogène, consiste en un mélange de séquences différentes. Finalement, ces dernières années, des séquences uniques ont pu être isolées grâce au clonage moléculaire.

\section{LES TECHNIQUES DU CLONAGE MOLÉCULAIRE}

Le clonage moléculaire consiste à joindre un morceau d'ADN à un transporteur moléculaire appelé vecteur qui sert à la propagation de cet ADN dans une bactérie réceptrice. Ce vecteur (plasmide, phage, cosmide) a la capacité de se répliquer dans la cellule-hôte permettant ainsi au fragment d'ADN étranger qui lui est attaché de se perpétuer indéfiniment. Une séquence unique d'ADN peut ainsi être isolée d'un génome complexe à partir d'une population de molécules d'ADN recombinées appelée bibliothèque ou banque génomique. Une banque est complète si le nombre de clones obtenu est suffisamment grand pour représenter la totalité de l'ADN chromosomique. Connaissant la longueur moyenne des fragments d'ADN clonés et la taille du génome haploïde, ce nombre peut être aisément calculé (CLARKE \& CARBON, 1976). Par exemple un génome de $3 \times 10^{6}$ kilopaires de bases $(\mathrm{kb})$ (tel que le génome des mammifères) cloné à partir de fragments de $15 \mathrm{~kb}$ en moyenne nécessite environ $10^{6}$ molécules recombinées pour atteindre une probabilité de 99 p. 100 d'obtenir une séquence donnée. Des banques complètes ont été créées à partir d'ADN chromosomique d'organismes très différents. Ce but a été atteint pour la première fois par MANIATIS et al. en 1978. Des banques partielles ont également été obtenues par enrichissement, avant le clonage, de séquences d'ADN particulières (BEDBROOK et al., 1980 ; GERLACH \& BEDBROOK, 1979).

Les bibliothèques d'ADN chromosomique peuvent être obtenues par 2 voies différentes mais complémentaires : clonage direct de l'ADN chromosomique ou clonage d'ADN complémentaire $(A D N c)$. La fabrication d'ADNc se fait par copie in vitro d'une popula- 
tion entière d'ARN messagers grâce à la transcriptase réserve (Williams, 1981). Dans cette $2^{\mathrm{e}}$ partie, les différents vecteurs utilisés en génie génétique seront décrits succinctement ; leurs avantages et leurs inconvénients seront résumés. Jusqu'à présent, ce sont les plasmides bactériens qui ont été le plus communément utilisés pour l'obtention de banques génomiques à partir de matériel végétal. Cependant, comme indiqué un peu plus loin, les vecteurs dérivés du bactériophage lambda et les cosmides offrent d'autres avantages. De toute façon, on ne doit pas oublier que les stratégies employées en génie génétique évoluent de façon très rapide.

\section{A. Clonage avec les plasmides}

Un plasmide bactérien est une molécule d'ADN double brin, circulaire, qui contient, suffisamment d'information génétique pour pourvoir à sa propre réplication ; il peut coder aussi pour des protéines qui confèrent à l'hôte bactérien une résistance à certains antibiotiques. Ces plasmides qui existent à l'état naturel ont été modifiés génétiquement afin de faciliter leur utilisation comme vecteurs de clonage. pBR32 est une molécule d'ADN de 4,362 pb (SUTCLIFFE, 1978) qui confère la résistance aux antibiotiques ampicilline (amp) et tétracycline (tet) (fig. 1 ; BOLIVAR et al., 1977 ; BOLIVAR \& BACKMAN, 1980). Cette molécule d'ADN peut être rendue linéaire, par exemple après traitement avec l'enzyme de restriction Ban $\mathrm{H} 1$ pour laquelle un site unique de coupure est présent dans le gène tet. Un fragment d'ADN étranger obtenu après hydrolyse par Ban $\mathrm{H} 1$ (ou un autre isoschizomère tel que Sau 3A) peut être inséré dans ce site et attaché de façon covalente avec la ligase T4 (PANASENKO et al., 1977), produisant ainsi un plasmide hybride ou recombiné. Cette molécule recombinante est alors introduite dans une bactérie (Escherichia coli) par transformation. Celle-ci est possible si les cellules bactériennes sont rendues compétentes par traitement au calcium (MANDEL \& HIGA, 1970). Afin d'éviter la possibilité de réarrangements de séquences d'ADN du plasmide hybride pendant l'amplification du nombre de copies, les molécules recombinées sont propagées dans une souche bactérienne qui est défectueuse pour la recombinaison: les souches $\operatorname{Rec} A$ - telles que HB101 ou ED8767 sont couramment employées. Les cellules bactériennes ainsi transformées peuvent être sélectionnées grâce à leur résistance en milieu ampicilline et testées pour leur sensibilité en milieu tétracycline puisque la résistance à cet antibiotique a été perdue lors de l'insertion du fragment d'ADN étranger dans le gène tet. Le nombre de copies de cette molécule recombinée peut alors être amplifié considérablement par culture des bactéries en milieu contenant un inhibiteur de synthèse protéique te!s que le chloramphenicol ou la spectinomycine (CLEWELL, 1972). Les plasmides dérivés de ColE1, pMB1 sont les plus utiles. Une dizaine de copies existent à l'état naturel et peuvent être amplifiées jusqu'à 1500 copies en milieu chloramphénicol. Une liste des vecteurs plasmides est présentée dans les publications de BERNARD \& HELSINKI (1980), BOLIVAR \& BACKMAN (1980) et THOMPSON (1982).

Afin d'accroître les chances d'insertion de fragments d'ADN d'origine diverse dans un vecteur donné,
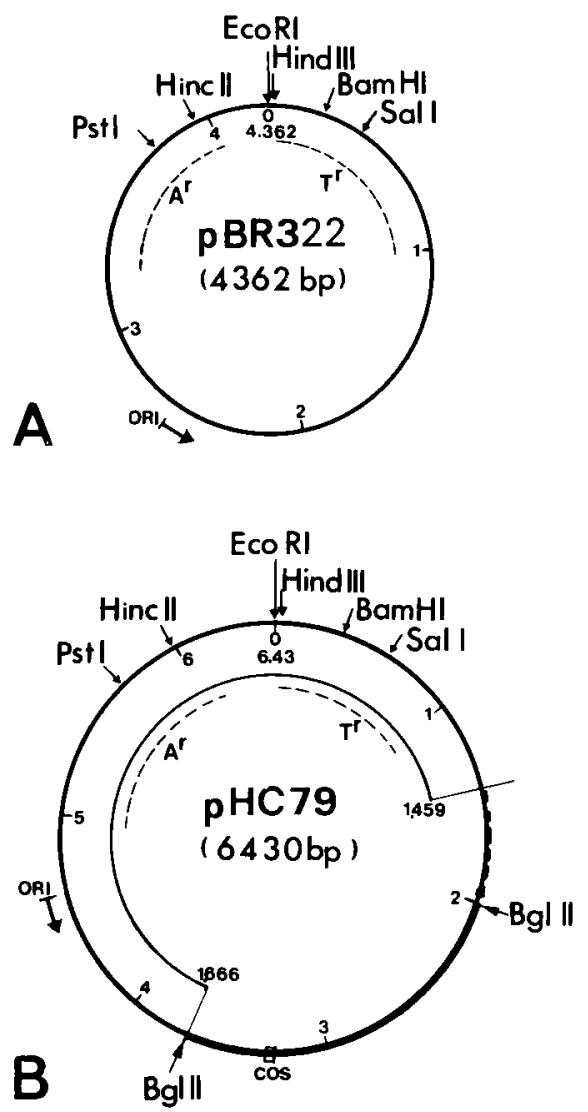

Figure 1

Représentation schématique des cartes physiques du plasmide pBR322 (A) et du cosmide pHC79 (B). Les tirets représentent les régions où sont localisés les gènes codant pour la résistance à l'ampicilline $(A r)$ et la tétracycline $(T)$. ORI représente l'origine et la flèche la direction de la réplication. Les cartes sont basées sur les publications de : (A) J. C. SUTCLIFFE, Nucl. Acids Res., 5, 2721 (1978) et (B) B. HOHN \& J. CollINS, Gene, 11, 291 (1980). Le cercle intérieur à pHC79 représente les séquences de pBR 322. La ligne épaisse représente le fragment BglII contenant les séquences cos, et les tirets un fragment du phage $\lambda$ de $0,65 \mathrm{~kb}$ également inséré dans pHC79. Le fragment comprenant les paires de base 1459-1666 de pBR322 a été perdu dans pHC79.

Schematic representation of the map of the plasmid pBR322 and the cosmid pHC79. Indicated in broken lines are the regions of genes encoding drug resistance : $A^{r}=$ ampicillin resistant, $T^{r}=$ tetracyclin resistant. ORI represents the origin of replication and the arrow the direction of replication. $A$ : the map is based on J. G. SuTCLIFFE, Nucl. Acids Res., 5, 2721 (1978). B : the map is based on B. HOHN \& J. COLLINS, Gene, II, 29I (1980). The inside circle of pHC79 represents the pBR322 sequences. The thick line represents the BgllI cos fragment and thick broken lines a $0.65 \mathrm{~kb}$ fragment of $\lambda$ phage also inserted in PHC79. The 1459 to $1666 \mathrm{pb}$ fragment of pBR322 has been deleted.

diverses stratégies expérimentales ont été développées. L'une consiste à modifier les termini d'un vecteur linéarisé par enzyme de restriction, afin d'éviter que les molécules vectrices non-attachées à un morceau d'ADN étranger se recircularisent entre elles ; sinon les ligations intramoléculaires seraient, de loin, l'évènement le plus fréquent et la sélection des clones nonrecombinés serait beaucoup trop élevée. La modification de ces extrémités consiste en l'élimination du phosphate 5' terminal par la phosphatase alcaline (UlLRICH et al., 1977). La recircularisation du plasmide n'est possible qu'après insertion d'ADN double brin possédant un groupe phosphate à ses extrémités 5' (WEAVER \& WEISSMAN, 1979). 
Afin de permettre l'utilisation d'un grand nombre d'enzymes de restriction pour un vecteur donné, 2 méthodes peuvent être utilisées: l'une consiste en l'addition d'un fragment d'ADN synthétique (SCHELLER et al., 1977 ; BAHL et al., 1977) au vecteur linéaire, par ligation bout franc (SGARAMELLA, 1972) ; ce fragment d'une dizaine de nucléotides, appelé « linker », possède une séquence qui peut être reconnue par une ou plusieurs enzymes de restriction ; la $2^{\mathrm{e}}$ méthode repose sur le principe d'hybridation des polynucléotides poly $d(A)$-poly $d(T)$ ou poly $d(G)$-poly $\mathrm{d}(\mathrm{C})$ qui ont été ajoutés aux extrémités du vecteur et de fragments d'ADN chromosomique grâce à la transférase terminale déoxynucléotidyle (WENSINK et al., 1974).

Après avoir obtenu un ensemble de clones (tel que la banque BamHI de pBR322), ceux-ci doivent être identifiés. L'identification de clones contenant une séquence recherchée se fait normalement en 2 étapes. Si une sonde moléculaire est disponible, la méthode d'hybridation moléculaire in situ de colonies (GRUNSTEIN \& HOGNESS, 1975) peut être employée. Cette méthode consiste à transférer les transformants sur papier de nitro-cellulose et à les amplifier sur milieu sélectif. Les colonies sont lysées in situ, l'ADN fixé au filtre, dénaturé, puis hybridé aux séquences de la sonde radioactive marquée au Phosphore 32 (SouTHERN, 1975). Ce premier criblage permet l'identification des clones qui sont alors examinés individuellement (analyse par enzymes de restriction, hybridation...) afin de déterminer si la séquence recherchée est présente. De tels clones peuvent alors être utilisés pour la méthode d'hybridation in situ décrite dans la $1^{\text {re }}$ partie de cet article (BEDBROOK et al., 1980 ; GERLACH et al., 1982).

\section{B. Clonage avec le phage lambda}

Un avantage du clonage avec le plage $\lambda$ est que son ADN peut être purifié facilement et en grande quantité. Il est possible d'obtenir $0,5 \mathrm{mg} / \mathrm{ml}$ d'ADN à partir de phages purifiés par centrifugation en gradient de chlorure de césium. L'efficacité avec laquelle un fragment particulier d'ADN peut être cloné dépend de la disponibilité d'un bon vecteur.

Deux découvertes importantes ont eu un grand impact sur l'utilisation de $\lambda$ en tant que vecteur. Tout d'abord l'observation qu'un tiers de la partie centrale $\mathrm{du}$ gènome de $\lambda$ ne contient que des gènes n'intervenant pas directement sur sa croissance lytique (fig. 2 ; HENDRIX, 1971). Cette région peut donc être remplacée par un morceau d'ADN étranger. L'autre observation découle de l'étude de la morphogenèse de $\lambda$ (revue par HOHN \& KATSURA, 1977) et elle a permis la mise au point de la technique d'encapsidation in vitro (ou packaging). L'ADN de $\lambda$, mis en présence de protéines d'encapsidation et de précurseurs nécessaires à la morphogenèse du phage, peut donner lieu à la production de particules infectieuses (BECKER \& GOLD, 1975). Le substrat pour l'encapsidation in vitro est une molécule concatémère d'ADN de $\lambda$ contenant les séquences cos (régions des extrémités cohésives de 2) (HOHN, 1975 ; FeIss et al., 1977 ; HOHN \& MURRAY, 1977 ; STERNBERG et al., 1977 ; HOHN, 1979) et seuls les monomères de longueur appropriée, soit 75 à 105 p. 100 de génome $\lambda$, peuvent produire des particu- les viables (WEIL et al., 1973). L'insertion dans le génome $\lambda$ de fragments d'ADN de plus de $22 \mathrm{~kb}$ n'est donc pas possible. L'encapsidation est un ensemble de réactions très complexes se produisant à la suite d'un mélange de 2 lysats bactériens obtenus à partir de souches lysogènes pour le phage $\lambda$ qui contiennent des prophages possédant des gènes mutés codant pour différentes étapes de la morphogenèse du phage. Lorsque ces 2 extraits sont mélangés avec l'ADN DE $\lambda$, ils se complémentent pour produire des particules infectieuses (HOHN \& MURRAY, 1977 ; STERNBERG et al., 1977).

\section{Les différents types de vecteurs lambda}

Un génome de $\lambda$ délété de sa partie non-essentielle et possédant un seul site de coupure pour une enzyme de restriction particulière est appelé un vecteur d'insertion (ce sont les plus utiles pour le clonage de petits fragments d'ADN, $10 \mathrm{~kb}$ ). Les vecteurs de remplacement sont ceux qui ont conservé 2 sites de coupure par une même enzyme de restriction, localisés aux extrémités de fragments d'ADN non-nécessaires au développement lytique de $\lambda$. Cette région nonessentielle, quand elle est remplacée par un fragment de taille appropriée, permet la formation de particules viables. Des fragments d'ADN de $6 \mathrm{~kb}$ à $20 \mathrm{~kb}$ peuvent être insérés (THOMAS et al., 1974 ; MURRAY \& MURRAY, 1975 ; MURRAY et al., 1977 ; BLATTNER et al., 1977 ; LOENEN \& BRAMMAR, 1980).

Les vecteurs dérivés de $\lambda$ les plus utilisés sont ceux qui permettent une insertion de fragments EcoRI, Hind III ou Ban HI (RIMM et al., 1980 ; POURCEL et al., 1979). Une liste de ces vecteurs a été établie par WILLIAMS \& BLATTNER (1980) et BRAMMAR (1982); ces auteurs ont aussi catalogué les sites de restriction et la longueur limite des fragments d'ADN qui peuvent être insérés.

\section{Identification et sélection des recombinants}

Le $1^{\text {er }}$ criblage qui identifie les recombinants à partir d'une grande population de colonies est basé sur des tests de couleur ou de changement de morphologie des plaques. Les plaques de phage $\lambda$ sauvage sont turbides, parce que certaines bactéries ont été lysées à la suite de l'infection. Les phages recombinants peuvent être reconnus par la formation de plaques claires (MURRAY \& MURRAY, 1975 ; BLATTNER et al., 1977 ; MURRAY et al., 1977 ; KLEIN \& MURRAY, 1979). Dans le cas des tests de couleurs, le phage vecteur produit des plaques bleues tandis que les phages recombinants produisent des plaques incolores (BLATTNER et al., 1977 ; POURCEL \& TIOLlaIs, 1977). D'autres tests, bien que plus laborieux, sont disponibles; ce sont les procédés de replique ou "spot tests " (MURRAY \& MURRAY, 1974).

Le $2^{\mathrm{e}}$ criblage se fait par hybridation lorsqu'une sonde moléculaire est disponible. Cette méthode est simplement une modification de la technique d'hybridation de colonies mise au point par GRUNSTEIN \& HOGNESS (Voir plasmides ; KRAMER et al., 1976 ; BENTON \& DAVIS, 1977). C'est une technique très sensible - des séquences de $1 \mathrm{~kb}$ ou moins peuvent être détectées - et très rapide puisque $10^{5}$ plaques peuvent être détectées sur une boîte de Petri de $9 \mathrm{~cm}$ de diamè- 

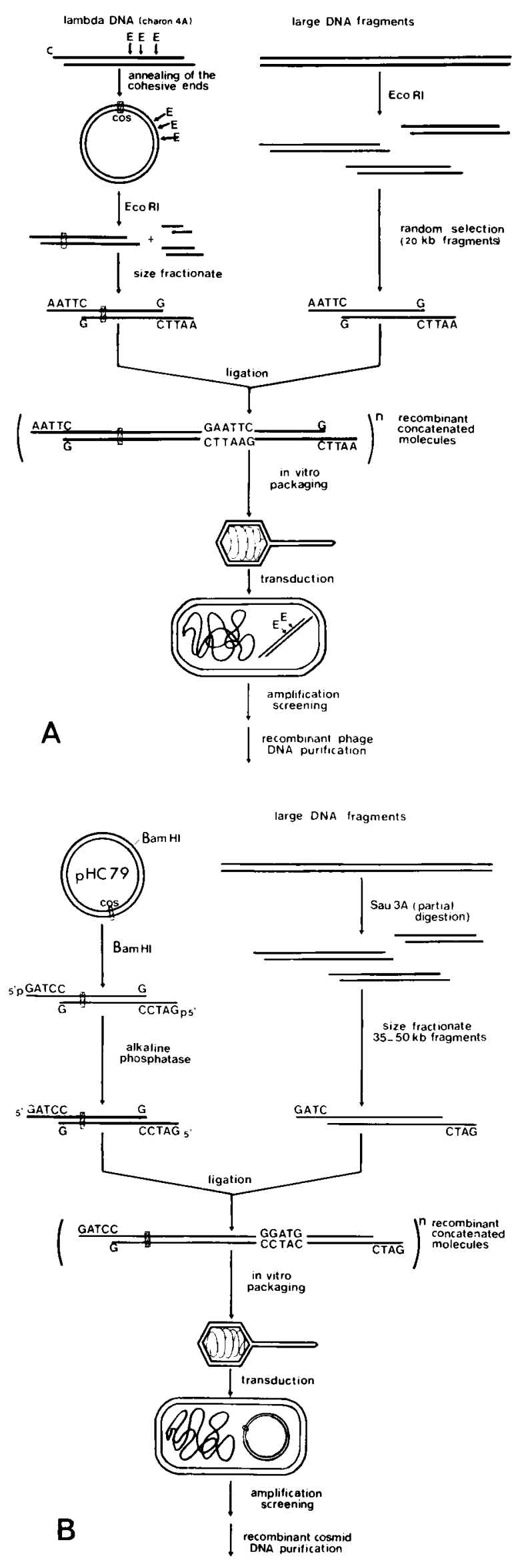

Figure 2

Diagramme schématique illustrant deux des stratégies utilisées pour la construction de banques génomiques avec comme vecteur le phage $\lambda(A)$ ou le cosmid pHC79 (B). L'ADN à haut poids moléculaire (ou $A D N C$ ) est d'abord fragmenté par hydrolyse partielle et les tre (JEFFREYS et al., 1982). La méthode d'obtention de banques génomiques à partir de $i$ se trouve détaillée dans la publication de DAHL et al. (1981).

\section{Comparaison des vecteurs : lambda-plasmides}

L'efficacité avec laquelle une bactérie est transformée par un plasmide dépend avant tout de la taille de celui-ci. De grands plasmides $(\simeq 50 \mathrm{~kb})$ transforment de 100 à 1000 fois moins que de petits plasmides ( $\simeq 5 \mathrm{~kb}$ ) (COLlins, 1977 ; COLlins \& HOHN, 1978).

L'encapsidation in vitro de $\lambda$ type sauvage produit $10^{8}$ plaques par g d' $\mathrm{ADN} \lambda$ et $5 \times 10^{4}$ à $5 \times 10^{5}$ plaques par g d'ADN recombiné, ce qui est de 100 à 1000 fois plus élevé que l'obtention de plasmides recombinés de taille identique (COLlins \& HOHN, 1978).

\section{Clonage avec les cosmides}

Afin de pouvoir cloner efficacement de grands fragments d'ADN, de nouveaux vecteurs appelés cosmides ont été développés (HOHN, 1975 ; COLLINS \& HOHN, 1978). Les cosmides possèdent à la fois les propriétés des plasmides et des phages et présentent des avantages par rapport aux deux. Ce sont des plasmides qui en plus contiennent les régions cohésives (cos) du bactériophage $\lambda$ (FEISS et al., 1977) permettant l'encapsidation in vitro (voir clonage avec $\lambda$ ). Un exemple d'obtention d'un vecteur cosmide est décrit par HOROWITZ et al. (1980).

Semblable à un plasmide, un vecteur cosmide présente un site unique de coupure par une ou plusieurs enzymes de restriction ; en outre, il possède une origine de réplication permettant une reproduction autonome dans la bactérie et il porte un marqueur sélectif tel que la résistance à certains antibiotiques permettant la sélection des recombinants. Semblables au phage $\lambda$, les cosmides contiennent les séquences cos nécessaires à l'encapsidation in vitro des molécules recombinées. L'encapsidation est possible seulement si les séquences cos sont séparées de 37 à $52 \mathrm{~kb}$, une distance qui correspond à 73 à $105 \mathrm{p}$. $100 \mathrm{du}$ génome $\lambda$ (voir clonage avec $\lambda$ ) (FEISS et al., 1977). Ainsi un vec-

fragments de longueur appropriée sélectionnée : 35 à $50 \mathrm{~kb}$ pour pHC79, $20 \mathrm{~kb}$ pour le phage $\lambda$, basé sur la stratégie utilisée par MANIATIS et al. (1978). La ligation du vecteur et du fragment d'ADN étranger se fait à haute concentration d'ADN afin de permettre la formation de longues molécules multimériques contenant les séquences cos. Les monomères linéaires de longueur appropriée peuvent alors être encapsidés in vitro (voir texte). La croissance des bactéries réceptrices au phage $\lambda$ et menée en fin de phase exponentielle dans un milieu $L+0,4 p .100$ maltose, puis les cellules sont concentrées dans 0,01 M MgSO 4 (HoHN \& CoLLINS, 1980). L'adsorption se fait à la température ambiante pendant $20 \mathrm{~min}$. Les cellules sont alors diluées dans le milieu L et incubées à $37^{\circ} \mathrm{C}$ pour permettre l'expression des gènes de résistance.

Schematic diagram illustrating a strategy used to construct libraries of random eucaryotic DNA fragments (A) using $\lambda$ cloning, (B) using cosmid cloning. The high molecular weight DNA (or cDNA) is fragmented by restriction enzyme partial digest then sizing the $D N A$ to be cloned : 35 to $50 \mathrm{~kb}$ for $\mathrm{pHC} 79$ cloning, $20 \mathrm{~kb}$ for this particular phage $\lambda$, based on the strategy used by MaNIATIS et al. (1979). To form long concatenated molecules with interspersed cos sequences, ligation of vector and insert DNA is performed at high concentration. Linear monomeric genome of appropriate length (see text) can be packaged in vitro. The $\lambda$-sensitive bacteria are grown in $L$ broth $+0.4 \%$ maltose and concentrated in $0.01 \mathrm{M} \mathrm{MgSO}_{4}$ (HoHN \& Collins, 1980). Adsorption is at room temperature for $20 \mathrm{~min}$. Then the cells are diluted in L broth, incubated at $37{ }^{\circ} \mathrm{C}$ to allow expression of the resistance genes. 
teur cosmide permettant l'insertion d'un fragment d'ADN chromosomique le plus grand possible doit être le plus petit possible puisque la longueur totale de l'ADN qui peut être cloné dans un cosmide est égale à la longueur de $\lambda(37-52 \mathrm{~kb})$ diminuée de la longueur $\mathrm{du}$ vecteur (un cosmide de $6 \mathrm{~kb}$ permet des insertions de fragments de 30 à $45 \mathrm{~kb}$ tandis qu'un cosmide de $10 \mathrm{~kb}$ permet des insertions de fragments de 25 à $40 \mathrm{~kb}$ ).

L'emploi des cosmides permet le clonage de grands morceaux d'ADN dans un plasmide, clonage difficile dans un plasmide vecteur classique du fait de l'efficacité très faible de transformation de $E$. coli ; en même temps, il permet la sélection directe de ces grands morceaux d'ADN puisque le vecteur trop petit et les petites molécules ne peuvent pas être encapsidés. Il faut donc, avant de commencer le clonage, sélectionner ces grands fragments d'ADN afin d'éviter toute incorporation simultanée de plusieurs petits fragments qui, insérés ensemble dans un même cosmide, pourraient constituer ainsi un arrangement nonauthentique de gènes : ex cosmide phC79 (fig. 1B, 2B ; HoHN \& COllins, 1980).

pHC79 est un génome de pBR322 dans lequel a été introduit un site de coupure par BgIII. Dans ce site, localisé dans une partie non-essentielle du plasmide, un fragment BglII qui contient les extrémités cohésives du phage $\lambda$ Charon 4A (BLATTNER et al., 1977) a été inséré. Le cosmide, rendu linéaire par l'enzyme de restriction Ban HI par exemple, est lié aux fragments Sau 3A (ou autre isoschizomère) d'ADN chromosomique. Comme pour le clonage avec $\lambda$, la technique d'encapsidation in vitro permet la production de particules phagiques à partir de ces molécules hybrides. Après la transduction d' $E$. coli, rendue possible après traitement au calcium (MANDEL \& HIGA, 1970), chaque molécule d'ADN recombinée se comporte comme un plasmide classique et contrôle sa propre réplication. Les transformants sont sélectionnés grâce à l'expression de leurs marqueurs de résistance aux antibiotiques (voir plasmide) ainsi exprimée dans les bactéries transformées.

En permettant le clonage de grands fragments d'ADN, cette technique présente des avantages évidents :

- Une banque génomique complète peut être obtenue avec un nombre relativement petit de clones. Par exemple une banque génomique construite à partir d'un génome nucléaire de mammifères avec des fragments d'en moyenne $40 \mathrm{~kb}$ nécessite environ $7 \times 10^{4}$ clones pour être complète avec une probabilité de 95 p. 100 d'obtenir une séquence donnée (à comparer avec $10^{6}$ clones qui seraient nécessaires si la taille moyenne des fragments clonés était de $15 \mathrm{~kb}$ ).

- Le fait de pouvoir isoler de grandes séquences d'ADN contiguës permet l'étude de groupes de liaison de gènes dans leur intégrité.

- Enfin, ceci devrait permettre l'étude des génomes complexes des plantes supérieures par hybridation in situ de régions spécifiques des chromosomes mitotiques.

\section{Autres vecteurs}

Des vecteurs permettant de propager des gènes dans d'autres bactéries, Bacillus subtilis, et dans la levure
Saccharomyces cerevisiae (HOHN \& HINNEN, 1980), ont aussi été développés mais leur emploi est encore limité. Sont également disponibles des vecteurs dérivés des virus M13 et fd. (BARNES, 1980).

\section{APPLICATION DE LA TECHNIQUE D'HYBRIDATION IN SITU AVEC DES SÉQUENCES CLONÉES}

L'application récente, dans le cas des chromosomes des plantes, de la technique d'hybridation in situ a déjà apporté beaucoup d'informations en ce qui concerne la structure, l'organisation ou l'identification des chromosomes. Jusqu'à présent, la plupart des études ont été menées sur des espèces appartenant à la famille des Gramineae. C'est pourquoi les exemples qui suivent ont été limité à ce groupe.

\section{A. Structure et organisation des chromosomes}

Des études biochimiques ont montré que la plupart des séquences de l'ADN répétitif sont mélangées entre elles et avec des séquences uniques (FLAVELL \& SMITH, 1976 ; HUTCHINSON et al., 1981). Corrélativement la détection de ces séquences par hybridation in situ montre qu'elles ne peuvent pas être localisées avec précision et qu'elles sont dispersées sur les chromosomes (FLAVELL et al., 1981; HUTCHINSON et al., 1981). Par contre, d'autres séquences, en particulier les séquences hautement répétitives, ont pu être localisées avec précision sur les chromosomes. Chez le blé, en utilisant l'ADN $\operatorname{Cot}^{-2}$ et l'ADN satellite, la plupart des séquences hautement répétitives ont été localisées sur 9 paires de chromosomes, souvent près du centromère, mais avec aussi quelques sites interstitiels (GERLACH et al., 1978 ; GERLACH \& PEACOCK, 1980 ; DENNIS et al., 1980 ; PEACOCK et al., 1981a). En utilisant des progéniteurs diploïdes et des lignées pourvues de marqueurs télocentriques, il a été possible d'identifier les 7 chromosomes du génome B et les séquences des chromosomes 4 et $7 \mathrm{du}$ génome $\mathrm{A}$. D'autres hybridations in situ faisant appel à des séquences clonées ont confirmé ces résultats (fig. $3 a$ ) et elles ont également permis de mettre en évidence d'autres séquences présentes sur les mêmes chromosomes (HUTCHINSON, 1981 ; HUTCHINSON \& LONSDALE, 1982). Des séquences clonées ont en outre été localisées dans les régions télomériques des chromosomes (fig. 3b). Chez le seigle, les séquences hautement répétitives ont été localisées, dans les zones télomériques ou aux extrémités des chromosomes, avec seulement quelques sites interstitiels sur certains chromosomes (APPELS et al., 1978 ; BEDBROOK et al., 1980 ; JONES, 1980 ; APPELS et al., 1981). Ces hybridations in situ montrent, comme dans le cas précédent, que chaque site d'hybridation permet d'identifier soit une seule, soit plusieurs familles de séquences répétitives.

Chez de nombreux animaux, une corrélation a pu être établie entre la localisation des séquences répétitites et la localisation de l'hétérochromatine constitutive. Chez le blé, des techniques de coloration ont montré que l'hétérochromatine est localisée pour l'essentiel sur le génome B et le chromosome 4A (GILL \& KIMBER, 1974 ; GERLACH, 1977). Une comparaison de la distribution de cette hétérochromatine 


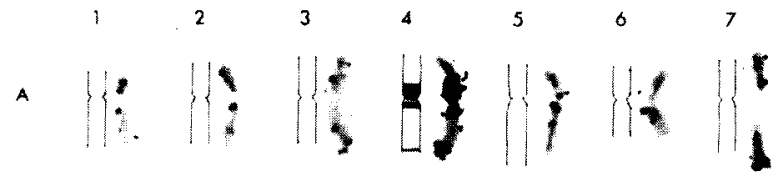

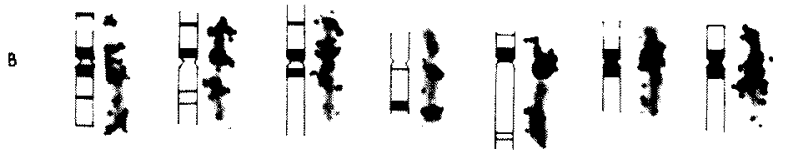

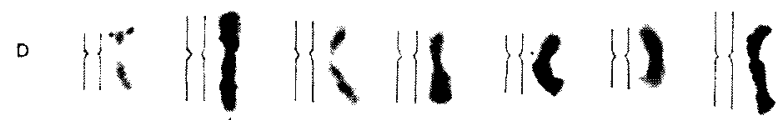

a)

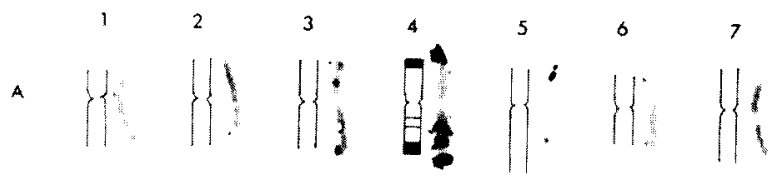

B

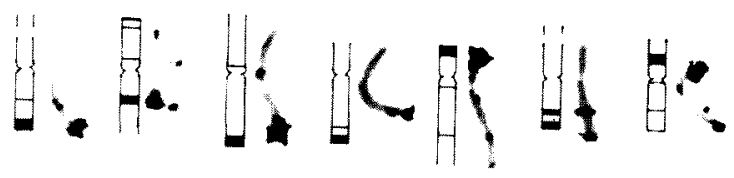

D

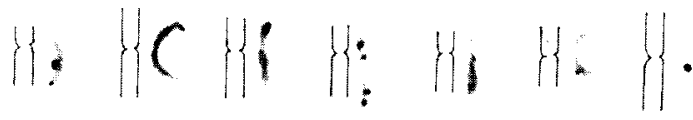

b)

Figure 3

3a. Hybridation in situ d'une copie d'ARNc (marquée avec ${ }^{3} \mathrm{H}$ ) de la séquence clonée TC22b et des chromosomes mitotiques de Triticum aestivum. Les chromosomes sont rangés en groupes homologues pour chaque génome $A, B$ et $D$.

3a. In situ hybridization of a ${ }^{3} \mathrm{H}$-labelled cRNA copy of cloned sequence TC22b with the mitotic chromosomes of Triticum aestivum. The chromosomes are arranged in homologous groups within the genomes $A, B$ and $D$.

3b. Hybridation in situ de la séquence hautement répétitive $C 40 \mathrm{du}$ blé et des chromosomes de $\mathrm{T}$. aestivum.

Les détails sur ces sondes se trouvent dans HUTCHINSON \& LONS DALE (1982).

3 b. In situ hybridization of the C40 sequence in wheat highly repetitive with chromosomes of $\mathrm{T}$. aestivum.

Details of both these probes are given in HUTCHINSON \& LONSDALE (1982).

avec la distribution des séquences répétitives a permis d'établir une corrélation (DENNIS et al., 1980 ; HUTCHINSON \& LONSDALE, 1982). Une telle relation a également été observée pour les séquences répétitives du seigle (APPELS et al., 1978 ; BEDBROOK et al., 1980 ; JONES, 1980). Dans ce dernier cas, la démonstration a été faite directement en hybridant tout d'abord les séquences répétitives du seigle aux chromosomes mitotiques, et ensuite en hybridant in situ la même préparation par la technique du C-banding (HUTCHINSON \& SEAL, 1983). Les mêmes corrélations se retrouvent aussi chez le maïs (PEACOCK et al., $1981 b$ ).

De telles séquences hautement répétitives ne semblent pas coder pour des protéines. Leur distribution, en parallèle à l'hétérochromatine telle que nous venons de la mentionner peut fournir une explication quant à l'inertie génétique de l'hétérochromatine comparée à l'euchromatine.

Un autre type de séquences répétitives qui sont au contraire transcrites sont celles porteuses des gènes d'ARN ribosomaux (ARNr). En utilisant la technique d'hybridation in situ, ces gènes d'ARNr ont été localisées chez Phaseolus (AVANZI et al., 1972 ; BRADY \& ClUTTER, 1972 ; DURANT et al., 1977), Vicia faba (SCHEUERMANN \& KNALMANN, 1975), Zea mays (PHILliPs et al., 1979), Hordeum vulgare (GERLACH \& BEDBROOK, 1979 ; APPELS et al., 1980) ainsi que chez différentes espèces de Triticum et Aegilops (GERLACH \& BEDBROOK, 1979 ; GERLACH et al., 1980 ; MILlER et al., 1980 ; CARROZZA, 1981 ; HUTCHINSON \& MILLER, 1982). La figure 4 montre une hybridation in situ typique de chromosomes mitotiques sondés avec des séquences clonées de gènes d'ARNr. Cette figure illustre bien la validité de la technique, en montrant premièrement les variations entre chromosomes du nombre de gènes d'ARNr présent dans chaque groupement (MILLER et al., 1980) et deuxièmement les variations de groupement de gènes d'ARNr entre différentes espèces, puisque les résultats issus de cette technique ne sont pas affectés par l'activité ou l'inac-

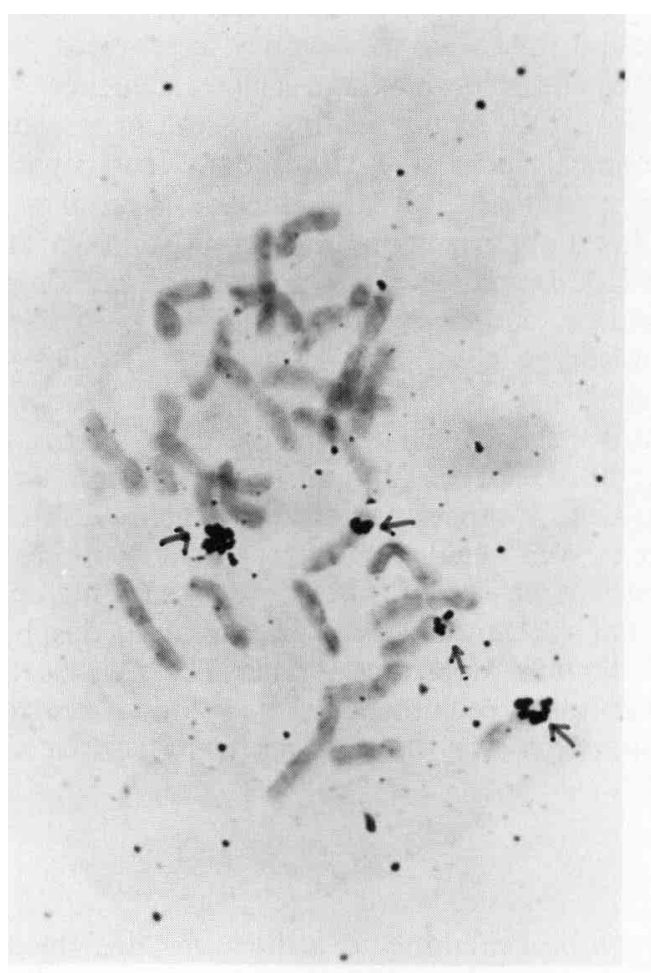

Figure 4

Hybridation in situ du gène cloné de l'ARN ribosomal et des chromosomes mitotiques de l'espèce tétraploïde Triticum araraticum. Deux paires de chromosomes ont été marqués par la sonde.

In situ hybridization of the cloned ribosomal RNA gene with mitotic chromosomes of the tetraploid species Triticum araraticum. Two pairs of chromosomes are labelled by the probe. 
tivité des gènes comme dans le cas d'autres techniques de coloration.

Toutes ces expériences utilisant comme sonde moléculaire soit les séquences de gènes d'ARNr, soit les séquences hautement répétitives, montrent comme il est possible d'utiliser la technique d'hybridation in situ pour étudier la structure, l'organisation et les variations entre chromosomes de plantes.

\section{B. Identification des chromosomes}

Une des propriétés de ces séquences hautement répétitives est leur localisation précise sur les chromosomes et sur certains chromosomes seulement (fig. $3 a$ et $3 b$ ). De plus, certaines de ces séquences sont présentes dans certaines espèces mais absentes (ou présentes en quantité non décelable) dans d'autres, et, par exemple, une séquence de $480 \mathrm{bp}$ présente à $1 \times 10^{6}$ copies par génome haploïde de Secale cereale ne peut être décelée chez le blé (BEDBROOK et al., 1980). En l'utilisant dans des expériences d'hybridation in situ, il a été possible de distinguer les chromosomes du seigle de ceux du blé dans les hybrides blé seigle (BREDBROOK et al., 1980). D'autres études ont également montré que cette séquence était absente chez Hordeum chilense et qu'il a été possible de reconnaître les chromosomes du seigle de ceux de cette orge dans les métaphases des mitoses de l'hybride $F_{1}$ tétraploïde entre ces 2 espèces (fig. $5 a$ ).

De plus, la technique d'hybridation in situ appliquée à d'autres stades que la métaphase a apporté des informations sur le comportement des chromosomes au cours de la mitose (fig. 5). La position des télomères de seigle a pu être identifiée tout au long de la mitose puisqu'ils sont marqués avec cette séquence répétitive de 480 bp. Cette même séquence a également été utilisée pour distinguer les chromosomes de $S$. cereale de ceux d'Aegilops dans leurs hybrides $\mathrm{F}_{1}$ soit à la mitose, soit à la méiose, ce qui a permis d'étudier l'appariement des chromosomes à la métaphase I de la méiose (HUTCHINSON et al., 1980).

D'autres séquences ont été utilisées pour l'étude de chromosomes à la méiose. En particulier la séquence $\mathrm{C} 40$, illustrée sur la figure $3 \mathrm{~b}$, a permis d'identifier un chromosome étranger d'Aegilops sharonensis chez le blé et d'en étudier l'appariement (MILlER et al., 1981). Les séquences porteuses des gènes d'ARN ribosomaux sont également très utiles pour l'étude d'appariement de chromosomes à la métaphase I. Elles ont été employées pour l'analyse des hybrides seigle Aegilops déjà mentionnée (HUTCHINSON et al., 1980) et plus récemment des hybrides $F_{1}$ entre Triticum timopheevi et $T$. dicoccum (HUTCHINSON et al., 1982).

\section{CONCLUSION}

On voit l'étendue d'utilisation des techniques d'hybridation in situ pour identifier les chromosomes des plantes. Cependant, jusqu'à présent leur emploi a été limité : on manque de séquences clonées et la plupart des études ont été faites sur un nombre limité d'espèces, principalement des céréales. Dans l'avenir, ces méthodes devraient permettre de localiser des gènes présents en exemplaire unique sur les chromoso-

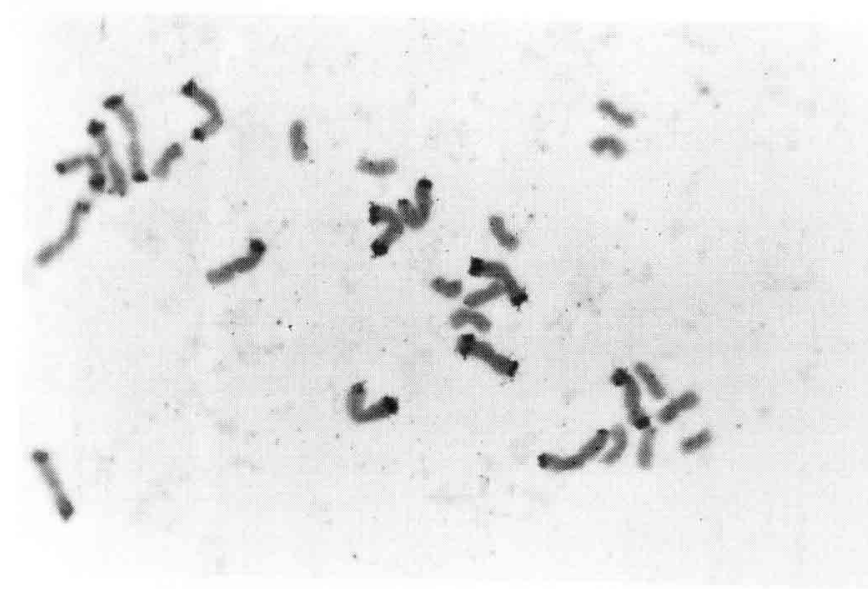

a)

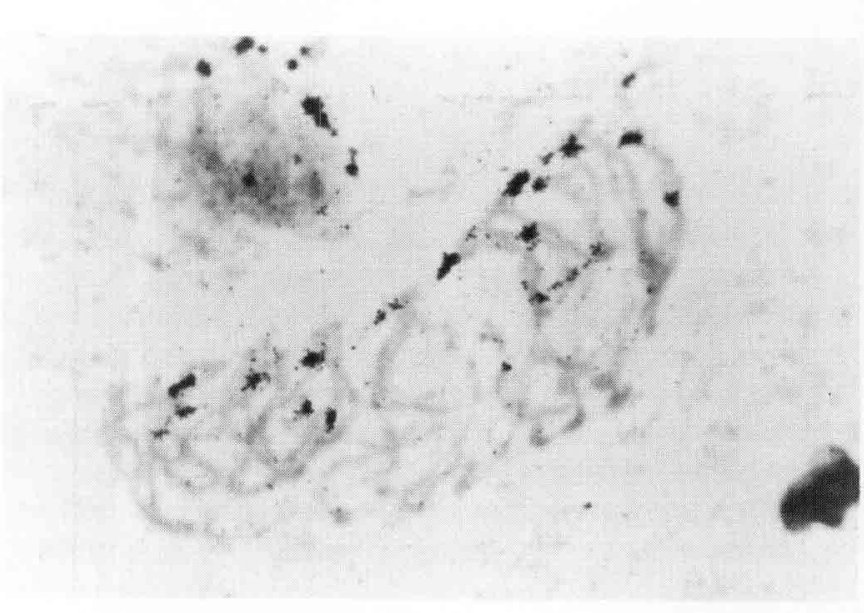

b)

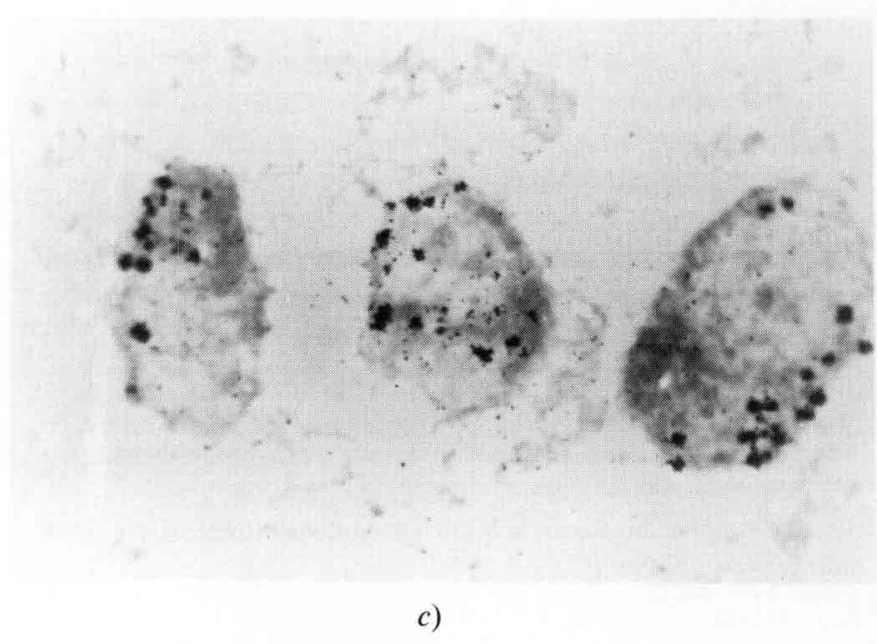

Figure 5

Hybridation in situ à différentes étapes de la mitose de la séquence de $480 \mathrm{bp}$ hautement répétitive du seigle et du noyau, d'un hybride tétraploïde entre Hordeum chilense et Secale cereale.

In situ hybridization of a cloned $480 \mathrm{pb}$ highly repetitive sequence from rye with the nucleus, at various stages of mitosis, of a tetraploid hybrid between Hordeum chilense and Secale cereale.

mes de plantes, comme on l'a déjà fait chez les animaux (RUDDLE, 1981) et on devra pouvoir conduire ces études chez un plus grand nombre d'espèces végétales. 


\section{RÉFÉRENCES BIBLIOGRAPHIQUES}

Appels R., Driscoll C., Peacock W. J., 1978. Heterochromatin and highly repeated DNA sequences in rye (Secale cereale). Chromosoma (Berl.), 70, 67-89.

Appels R., Gerlach W. L., Dennis E., Swift H., Peacock W. J., 1980. Molecular and chromosomal organization of DNA sequences in coding for the ribosomal RNAs in cereals. Chromosoma (Berl.), 78, 283-311.

Appels R., Dennis E. S., Smyth D. R., Peacock W. J., 1981. Two repeated DNA sequences from heterochromatic regions of rye (Secale cereale) chromosomes. Chromosoma (Berl.), 84, 265-277.

Avanzi S., Durante M., Cionini P. G., D'Amato F., 1972. Cytological localization of ribosomal cistrons in polytene chromosomes of Phaseolus coccineus. Chromosoma (Berl.), 39, 191-203.

Bahl C. P., Marians K. J., Wu R., Stawinsky J., Narang S., 1977. A general method for inserting specific DNA sequences into cloning vehicles. Gene, 1, 81 .

Barnes W. M., 1980. DNA cloning with single-stranded phage vectors. In 'Genetic Engineering'. Eds. J. K. Setlow \& A. Hollaender, Vol. 2, 185-200, Plenum Press, New York.

Becker A., Gold M., 1975. Isolation of the bacteriophage lambda A-gene protein. Proc. Nat. Acad. Sci., 72, 581-585.

Bedbrook J. R., Jones J., O'Dell M., Thompson R. D., Flavell R.B., 1980. A molecular description of telomeric heterochromatin in Secale species. Cell, 19, 545-560.

Benton W. D., Davis R. W., Screening $\lambda \mathrm{gt}$ recombinant clones by hybridization to single plaques in situ. Science, 196, 180.

Bernard H. U., Helinski D. R., 1980. Bacterial plasmid cloning vehicles. In 'Genetic Engineering'. Eds J. K. Setlow \& A. Hollaender, Vol. 2, 133-167. Plenum Press, New York.

Blattner F. R., Williams B. G., Blechl A. E., Denniston-Thompson K., Faber H. E., Furlong L., Grunwald J. D., Kiefer D. O., Moore D. D., Schumm J. W., Sheldon E. L., Smithies O., 1977. Charon phages : safer derivatives of bacteriophage lambda for DNA cloning. Science, 196, 161.

Bolivar F., Rodriguez R. L., Betlach M. C., Boyer H. W., 1977. Construction and characterization of new cloning vehicles. I. Ampicillin-resistance derivatives of the plasmid pMB9. Gene, 2, 7593.

Bolivar F., Backman K., 1980. Plasmids of Escherichia coli as cloning vectors. In 'Methods in Enzymology'. Ed. R. Wu, Vol. 68, 245-269. Academic Press, New York.

Brady T., Clutter M. E., 1972. Cytolocalization of ribosoma cistrons in plant polytene chromosomes. J. Cell. Biol., 53, 827-832.

Brammar W. J., 1982. Vectors based on bacteriophage lambda. In 'Genetic Engineering'. Ed. R. Williamson, Vol. 3, 53-81. Academic Press, New York.

Carrozza M. L., 1981. Number of sites for ribosomal RNA genes in Triticum durum. In situ hybridisation on mitotic, meiotic and polytene nuclei. $Z$. Pfz., 87, 300-308.

Clarke L., Carbon J., 1976. A colony bank containing synthetic ColEl hybrid plasmids representative of the entire $E$. coli genome. Cell, 9, 91 .

Clewell D. B., 1972. Nature of $\mathrm{ColE}_{1}$ plasmid replication in Escherichia coli in the presence of chloramphenicol. J. Bacteriol., $110,667-676$

Collins J., 1977. Gene cloning with small plasmids. Curr. Topics Microbiol. Immunol., 78, 121-170.

Collins J., John B., 1978. Cosmids : a type of plasmid genecloning vector that is packageable in vitro in bacteriophage $\lambda$ heads. Proc. Nat. Acad. Sci., 75, 4242-4247.

Dahl H. H., Flavell R. A., Grosveld F. G., 1981. The use of genomic libraries for the isolation and study of eukaryotic genes. In 'Genetic Engineering'. Ed. R. Williamson, Vol. 2, 49-127. Academic Press, New York.

Dennis E. S., Gerlach W. L., Peacock W. J., 1980. Identical polypyrimidine-polypurine satellite DNAs in wheat and barley. Heredity, 44, 349-366.

Deumling B., Greilhuber J., 1982. Characterization of heterochromatin in different species of the Scilla sibirica group (Liliaceae) by in situ hybridisation of satellite DNAs and fluorochrome banding. Chromosoma (Berl), 84, 535-555.
Durant M., Cionini P. G., Avanzi S., Cremonini R., D'Amato F., 1977. Cytological localization of the genes for the four classes of ribosomal RNA (25S, 18S, 5-8S, and 5S) in polytene chromosomes of Phaseolus coccineus. Chromosoma (Berl.), 60, 269-282.

Feiss M., Crayton R. A., Egner C., 1977. Packaging of bacteriophage 1 chromosome : effect of chromosome length. Virology, 77, 271-193.

Flavell R., 1980. The molecular characterization and organization of plant chromosomal DNA sequences. Annu. Rev. Plant Physiol., $31,569-596$

Flavell R. B., Smith D. B., 1976. Nucleotide sequence organisation in the wheat genome. Heredity, 37, 231-252.

Flavell R. B., O'Dell M., Hutchinson J., 1981. Nucleotide sequence organization in plant chromosomes and evidence for sequence translocation during evolution. Cold Spring Harbor Symp. Quant. Biol., 45, 501-508.

Gall J. G., Pardue M. L., 1971. Nucleic acid hybridisation in cytological preparations. In 'Methods in Enzymology'. Eds. L. Grossman \& K. Moldave. Academic Press, New York, NY. Vol. 21, 470-480

Gerlach W. L., 1977. N-banded karyotypes of wheat species. Chromosoma (Berl.), 62, 49-56.

Gerlach W. L., Appels R., Dennis E. S., Peacock W. J., 1978 Evolution and analysis of wheat genomes using highly repeated DNA sequences. Proc. 5th Int. Wheat Genet. Symp. Ed. S. Ramanujam, Vol. 1, 81-91.

Gerlach W. L., Bedbrook J. R., 1979. Cloning and characterization of ribosomal RNA genes from wheat and barley. Nucleic Acids Res., 7, 1869-1886.

Gerlach W. L., Peacock W. J., 1980. Chromosomal locations of highly repeated DNA sequences in wheat. Heredity, 44, 269-276,

Gerlach W. L., Miller T. E., Flavell R. B., 1980. The nucleolus organizers of diploid wheats revealed by in situ hybridisation. Theor. Appl. Genet., 58, 97-100.

Gerlach W. L., Dennis E. S., Peacock W. J., 1982. Molecular cytogenetics of wheat. In 'Cytogenetics of crop plants'. Eds. M. S. Swaminathan, P. K. Gupta, U. Sinha (in press).

Gill B. S., Kimber G., 1974. Giemsa C-banding and the evolution of wheat. Proc. Nat. Acad. Sci. U.S.A., 71, 4086-4090.

Grunstein M., Hogness D., 1975. Colony hybridization : a method for the isolation of cloned DNAs that contain a specific gene. Proc. Nat. Acad. Sci. U.S.A., 72, 3961-3965.

Hendrix R. W., 1971. In 'The Bacteriophage Lambda'. Ed. A.D. Hershey, 355-370. Cold Spring Harbor Laboratory.

Hohn B., 1975. DNA as a substrate for packaging in bacteriophage lambda. J. Mol. Biol., 98, 93-106.

Hohn B., 1979. In vitro packaging of 1 and cosmid DNA. In 'Methods in Enzymology', ed. R. Wu, 68, 299-309. Ac. Press N. Y.

Hohn T., Katsura K., 1977. In 'Current Tropics in Microbiology and Immunology', Vol. 78, 69-110.

Hohn B., Murray K., 1977. Packaging recombinant DNA molecules into bacteriophage particles in vitro. Proc. Nat. Acad. Sci., 74, 3259.

Hohn B., Collins J., 1980. A small cosmid for efficient cloning of large DNA fragments. Gene, 11, 291-298.

Hohn B., Hinnen A., 1980. Cloning with cosmids in E. coli and yeast. In "Genetic Engineering », Eds. J. K. Setlow \& A. Hollaender, Vol. 2, 169-183. Plenum Press, New York.

Horowitz M., Guild G. M., Prestidge L. S., Hogness D. S., 1980. A new high capacity cosmid vector and its use. Gene, 11, 271-282.

Hutchinson J., 1981. The identification of wheat chromosomes by means of the technique of in situ hybridisation of cloned DNA sequences. In "Induced Variability in Plant Breeding ". Proc. Eucarpia, PUDOC, Wageningen, The Netherlands, 42-44.

Hutchinson J., Chapman W., Miller T. E., 1980. Chromosome pairing at meiosis in hybrids between Aegilops and Secale species: A study by in situ hybridisation using cloned DNA. Heredity, 45, 245-254.

Hutchinson J., Flavell R. B., Jones J., 1981. Physical mapping of plant chromosomes by in situ hybridisation. In "Genetic Engi- 
neering ", Eds. J. K. Setlow \& A. Hollaender. Plenum Publishing Co., New York, NY, Vol. 3, 207-222.

Hutchinson J., Lonsdale D. M., 1982. The chromosomal distribution of cloned highly repetitive sequences from hexaploid wheat. Heredity, 48, 373-378.

Hutchinson J., Miller T. E., 1982. The nucleolar organisers of tetraploid and hexaploid wheats revealed by in situ hybridisation. Theor. Appl. Genet., 61, 285-288.

Hutchinson J., Seal A. G., 1983. A sequential in situ hybridisation and C-banding technique. Heredity, 51 (2), 507-509.

Hutchinson J., Miller T. E., Jahier J., Shepherd K., 1982. Comparison of the chromosomes of Triticum timopheevi with related wheats using the techniques of $\mathrm{C}$-banding and in situ hybridisation. Theor. Appl. Genet., 64, 31-40.

Jeffreys A. J., Barrie P. A., Harris S., Fawcett D. H., Nugent Z. J., Boyd A. C., 1982. Isolation and sequence analysis of a hybrid $\delta$-globin pseudogene from the Brown lemur. J. Mol. Biol., 156, 487-503.

Jones K. W., 1973. The method of in situ hybridization. In « New Techniques in Biophysics and Cell Biology », Eds R. H. Pain \& B. J. Smith, John Willey and Sons Ltd., New York and London, Vol. 1, 29-66.

Jones J., 1980. Repeated DNA sequences in rye (Secale cereale), wheat (Triticum aestivum) and their relatives. $\mathrm{Ph}$. $\mathrm{D}$. Thesis, University of Cambridge, UK.

Klein B., Murray K., 1979. Phage lambda receptor chromosomes for DNA fragments made with restriction endonuclease I of Bacillus amyloliquefaciens H. J. Mol. Biol., 133, 289.

Kramer R. A., Cameron J. R., Davis R., 1976. Isolation of bacteriophage $\lambda$ containing yeast ribosomal RNA genes : screening by in situ RNA hybridization to plaques. Cell, 8, 227-232.

Loenen W. A. M., Brammar W. J., 1980. A bacteriophage lambda vector for cloning large DNA fragments made with several restriction enzymes. Gene, 10, 249-259.

Mandel M., Higa A., 1970. Calcium-dependent bacteriophage DNA infection. J. Mol. Biol., 53, 159-162. Gene, 10, 249.

Maniatis T., Hardison R. L., Lacy R., Lauer J., O'Connell C., Quon D., Sim G. K., Efstradiadis A., 1978. Isolation of structural genes from libraries of eukaryotic DNA. Cell, 15, 687.

Miller T. E., Gerlach W. L., Flavell R. B., 1980. Nucleolus organiser variation in wheat and rye revealed by in situ hybridisation. Heredity, 45, 377-382.

Miller T. E., Hutchinson J., Chapman V., 1981. Investigation of a preferentially transmitted Aegilops sharonensis chromosome in wheat. Theor. Appl. Genet., 61, 37-33.

Murray N. E., Murray K., 1974. Manipulations of restriction targets in phage $\lambda$ to form receptor chromosomes for DNA fragments, Nature, 251, 331.

Murray K., Murray N. E., 1975. Phage lambda receptor chromosomes for DNA fragments made with restriction endonuclease III of Haemophilus influenzae and restriction endonuclease I of Escherichia coli. J. Mol. Biol., 98, 551.

Murray N. E., Brammar W. J., Murray K., 1977. Lambdoid phages that simplify the recovery of in vitro recombinants. Mol. Gen. Genet., 150, 53.

Panasenko S. M., Cameron J., Davis R. W., Lehman I. R., 1977. Five hundred fold over-production of DNA ligase after induction of a hybrid lambda lysogen constructed in vitro. Science, 196, 188-189.

Pardue M. L., Gall J. G., 1975. Nucleic acid hybridization to the DNA of cytological preparations. Meth. Cell. Biol., 10, 1-16.

Peacock W. J., Lohe A. R., Gerlach W. L., Dunsmuit P., Dennis E. S., Appels R., 1977. Fine structure and evolution of DNA in heterochromatin. Cold Spring Harbor Symp. Quant. Biol., 42, 1121-1135.

Peacock W. J., Gerlach W. L., Dennis E. S., 1981a. Molecular aspects of wheat evolution : Repeated DNA sequences, 41-50. In "Wheat science-today and tomorrow », Eds. L. T. Evans \& W. J. Peacock. Cambridge University Press, UK.

Peacock W. J., Dennis E. S., Rhoades M. M., Prior A. J., $1981 b$. Highly repeated DNA sequence limited to knob heterochromatin in maize. Proc. Nat. Acad. Sci. U.S.A., 78, 4490-4494.

Phillips R. L., Wang A. S., Rubenstein I., Park W. D., 1979.
Hybridization of ribosomal RNA to maize chromosomes. Maydica, 24, 7-32.

Pourcel C., Tiollais P., 1977. $\lambda$ plac 5 derivatives, potential vectors for DNA fragments cleaved by Sst I. Gene, 1, 281.

Pourcel C., Marchal A., Loise A., Fritsch A., Tiollais P., 1979. Bacteriophage lambda - E. coli K12 vector, host system for cloning and expressions under lactose promoter control. I. DNA insertion at the lacZ Eco RI restriction site. Mol. Gen. Genet., 170, 161 .

Ray J. H., Venketeswaran S., 1979. DNA replication, ${ }^{3}$ H-cRNA in situ hybridisation and C-band patterns in the polycentric chromosomes of Luzula purpurea Link. Chromosoma (Berl.), 74, 337-346.

Rimm D. L., Horness D., Huicera J., Blattner F. R., 1980. Construction of coliphage lambda Charon vectors with $B a m \mathrm{HI}$ cloning sites. Gene, 12, 301.

Ruddle F. H., 1981. A new era in mammalian gene mapping : somatic cell genetics and recombinant DNA methodologies. Nature, 294, $115-120$.

Scheller R. H., Dickerson R. E., Boyer H. W., Riggs A. D. Itakura K., 1977. Chemical synthesis of restriction enzyme recognition sites useful for cloning. Science, 196, 177.

Scheuermann W., Knalmann M., 1975. Localization of ribosomal cistrons in metaphase chromosomes in Vicia faba L. Exp. Cell Res., 90, 463-465

Sgaramella V., 1972. Enzymatic oligomerisation of bacteriophage P22 DNA and of linear Simian Virus 40 DNA. Proc. Nat. Acad. Sci., 69, 3389

Southern E. M., 1975. Detection of specific sequences among DNA fragments separated by gel electrophoresis. J. Mol. Biol., 98, 503.

Sternberg N., Tiemeier D., Enquist L., 1977. In vitro packaging of a Dam vector containing EcoRI DNA fragments of Escherichia coli and phage P1. Gene, 1, 255.

Sutcliffe J. G., 1978. Complete nucleotide sequence of the Escherichia coli plasmid pBR 322. Cold Spring Harb. Symp. Quant. Biol., 43, 77-90.

Szabo P., Elder R., Steffensen D. M., Uhlenbeck O. C., 1977. Quantitative in situ hybridisation of ribosomal RNA species to polytene chromosomes of Drosophila melanogaster. J. Mol. Biol., 115, 539-563.

Thomas M., Cameron J. R., Davis R. W., 1974. Viable molecular hybrids of bacteriophage $\lambda$ and eukaryotic DNA. Proc. Natl. Acad. Sci. U.S.A., 71, 4579-4583.

Thompson R., 1982. Plasmid and phage M13 cloning vehicles. In "Genetic Engineering », Ed. R. Williamson, Vol. 3, 2-41. Academic Press, New York.

Timmis J. N., Deumling B., Ingle J., 1975. Localisation of satellite DNA sequences in nuclei and chromosomes of two plants. Nature, 257, 152-155.

Ullrich A., Shine J., Chirgwin J., Pictet R., Tischer E., Rutrer W. J., Goodman H. M., 1977. Rat insulin genes : construction of plasmids containing the coding sequences. Science, 196, 1313-1319.

Weaver R. F., Weissmann C., 1979. Mapping of RNA by a modification of the Berk-Sharp procedure : the 5 ' termini of $15 \mathrm{~S}$ $\beta$-globin mRNA precursor and mature $10 \mathrm{~S} \beta$-globin mRNA have identical map coordinates. Nucl. Acids Res., 6, 1175-1193.

Weil J., Cunningham R., Martin R., Mitchell B., Bolling B., 1973. Characteristics of $\lambda \mathrm{p} 4$, a $\lambda$ derivative containing $9 \%$ excess DNA. Virology, 50, 373.

Wensink P. C., Finnegan D. J., Donelson J. E., Hogness D. S., 1974. A system for mapping DNA sequences in the chromosomes of Drosophila melanogaster. Cell, 3, 315.

Williams J. G., 1981. The preparation and screening of a cDNA clone bank. In « Genetic Engineering 2, Ed. R. Williamson, Vol. 1, 2-55. Academic Press, New York.

Williams B. G., Blattner F. R., 1980. Bacteriophage lambda vectors for DNA cloning. In « Genetic Engineering », Eds. J. K. Setlow \& A. Mullaender, Vol. 2, Plenum, N. Y.

Wimber D. E., Steffensen D. M., 1973. Localization of gene function. Annu. Rev. Genetics, 7, 205-223.

Wobus U., 1976. Hybridisierung von Nucleinsauren in situ. Biol. Zbl., 95, 1-24. 